Supporting information for:

\title{
Toward the Development of Ultrasensitive Detectors for Environmental Applications: A kinetic Study of Cr(III) monitoring in water Using EDTA and SERS Technique
}

Hassan Traboulsi*a and Chawki Awada*b

aDepartment of Chemistry, College of Science, King Faisal University, P.O Box: 400 Al-Ahsa, 31982, Saudi Arabia.

bepartment of Physics, College of Science, King Faisal University, P.O Box: 400 Al-Ahsa, 31982, Saudi Arabia.

Corresponding authors:

Hassan Traboulsi : Email: htraboulsi@kfu.edu.sa, Tel: +966135897404

Chawki Awada : Email: cawada@kfu.edu.sa, Tel : +966135899533 


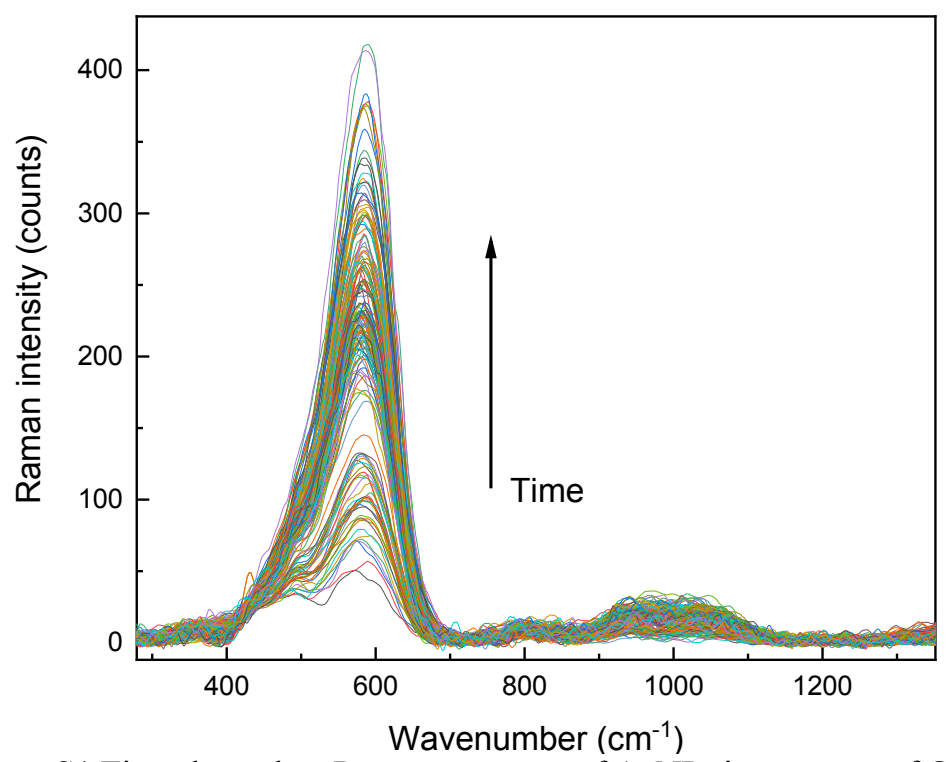

Figure S1 Time dependant Raman spectrum of AgNPs in presence of Cr(III).
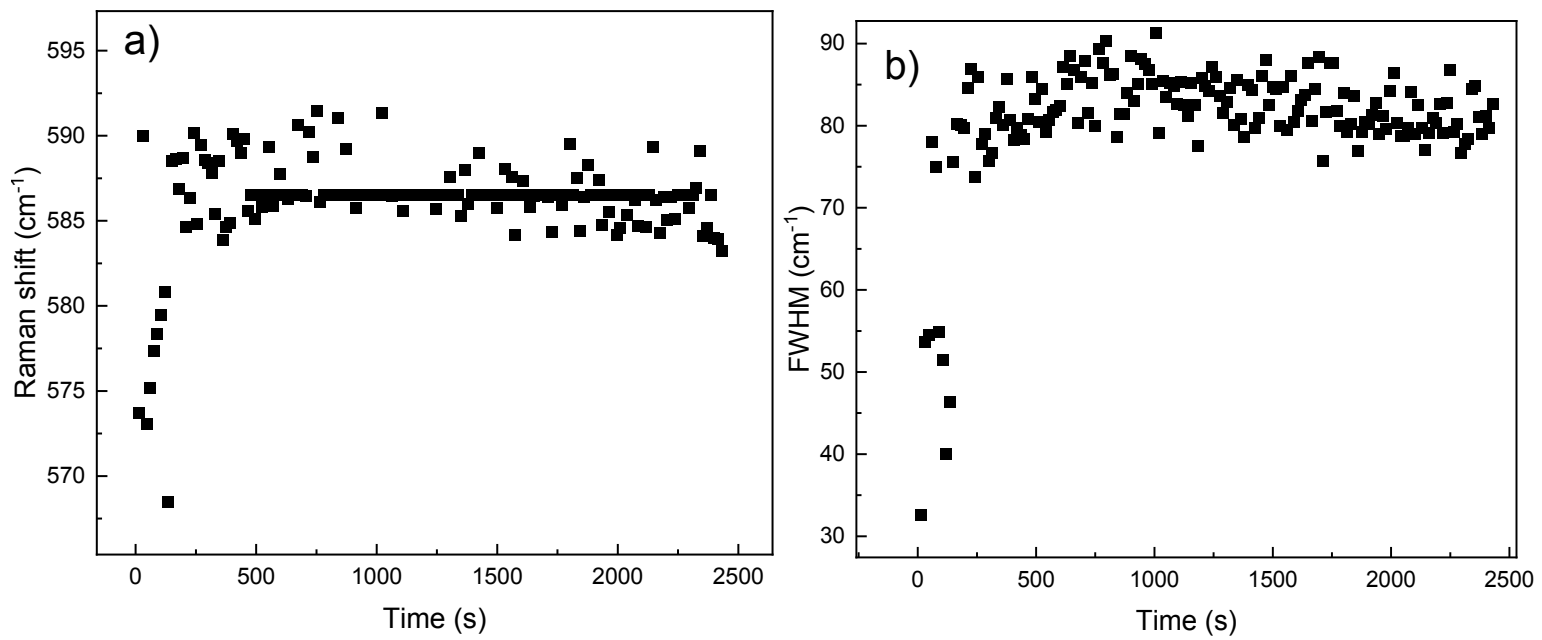

Figure S2 (a) Raman Shift versus time of $\mathrm{Cr}(\mathrm{III})$ in presence of AgNPs. (b) FWHM of the Raman peak of $\mathrm{Cr}(\mathrm{III})$ in presence of AgNPs.
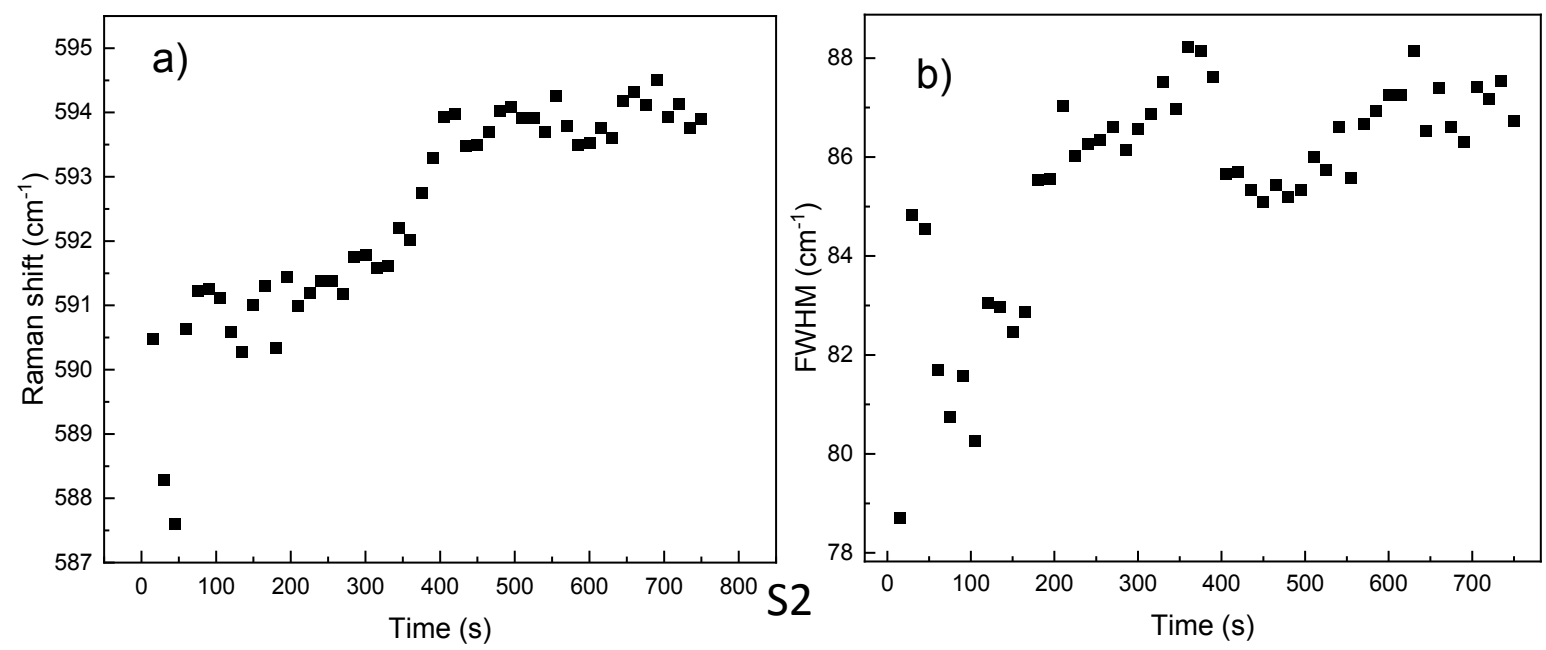
Figure S3 (a) Raman Shift versus time of Cr(III) in presence of AgNPs after adding EDTA. (b) FWHM of the Raman peak versus time of $\mathrm{Cr}(\mathrm{III})$ in presence of AgNPs after adding EDTA.

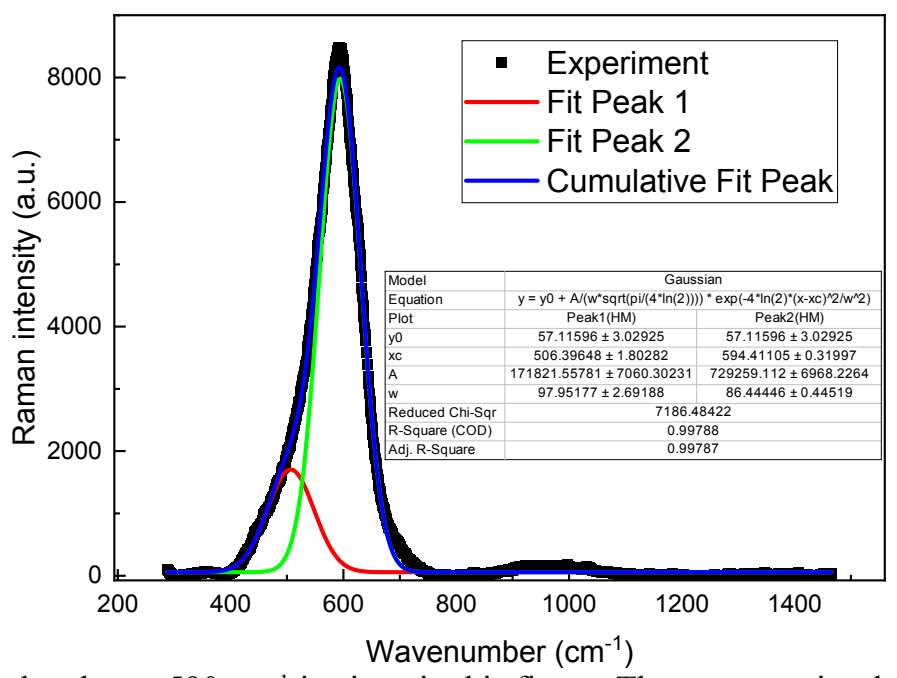

Figure S4 A fit of Raman band near $590 \mathrm{~cm}^{-1}$ is given in this figure. The spectrum is taken from Fig. 5 (a). For an excellent fit of the band of interest, we performed a deconvolution of the spectrum with a Gaussian function.

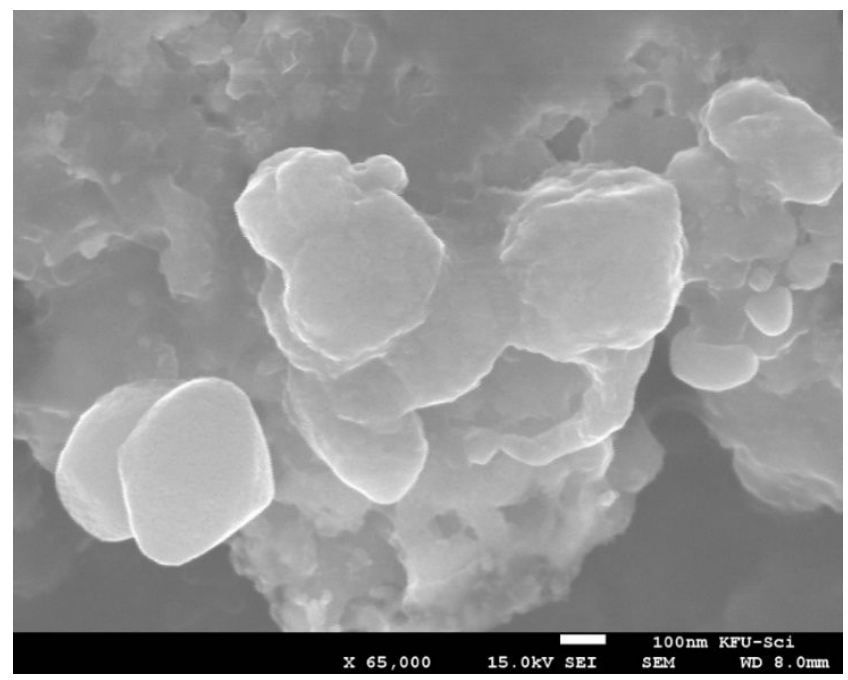

Figure S5 SEM image of AgNPs + Cr(III) + EDTA. The mixture was prepared 24 hours prior to sample deposition on a pure copper metal substrate disc. Two drops of the mixture were kept for water evaporation before performing the SEM measurements. 

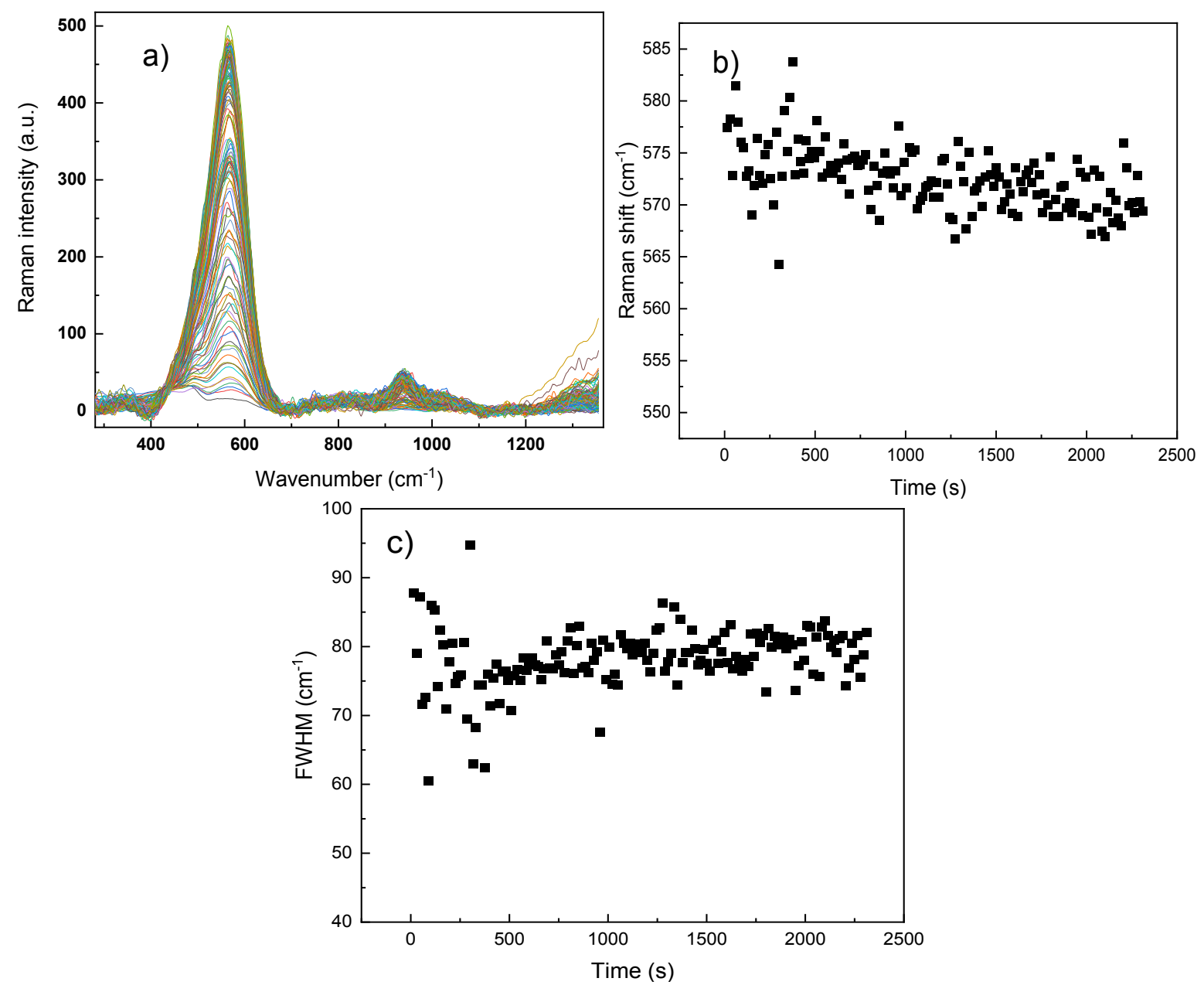

Figure S6 (a) Time dependant Raman spectrum of [Cr(EDTA) ${ }^{-}$in presence of AgNPs. (b) Raman Shift versus time of [Cr(EDTA) $]^{-}$in presence of AgNPs (c) FWHM of the Raman peak versus time of [Cr(EDTA) $]^{-}$in presence of AgNPs. 


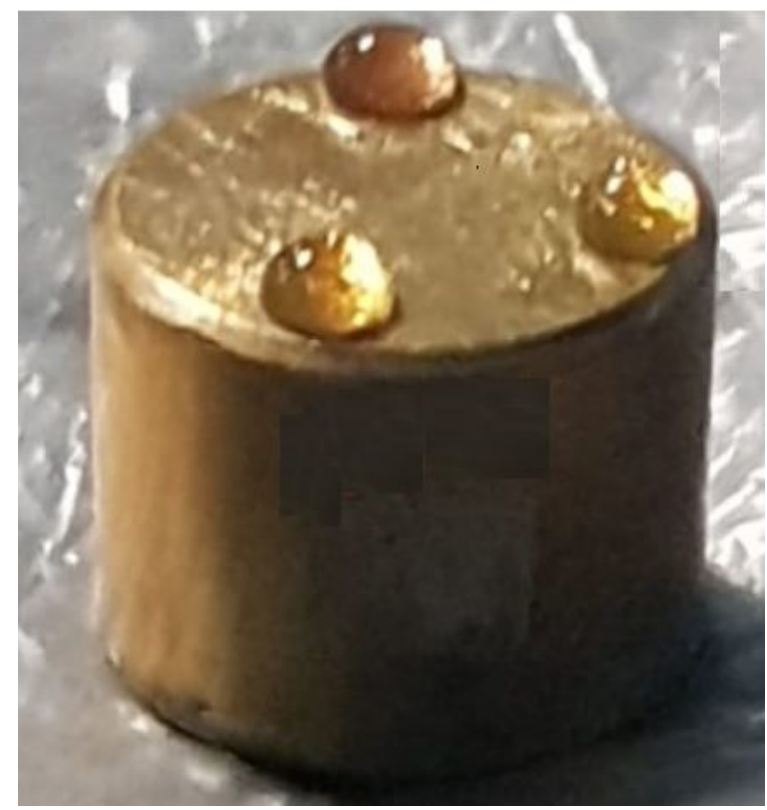

Figure S7 Deposition of the samples on the copper metal substrate disc. The drops were kept for water evaporation before performing the SEM measurements. The photo was taken by the authors.

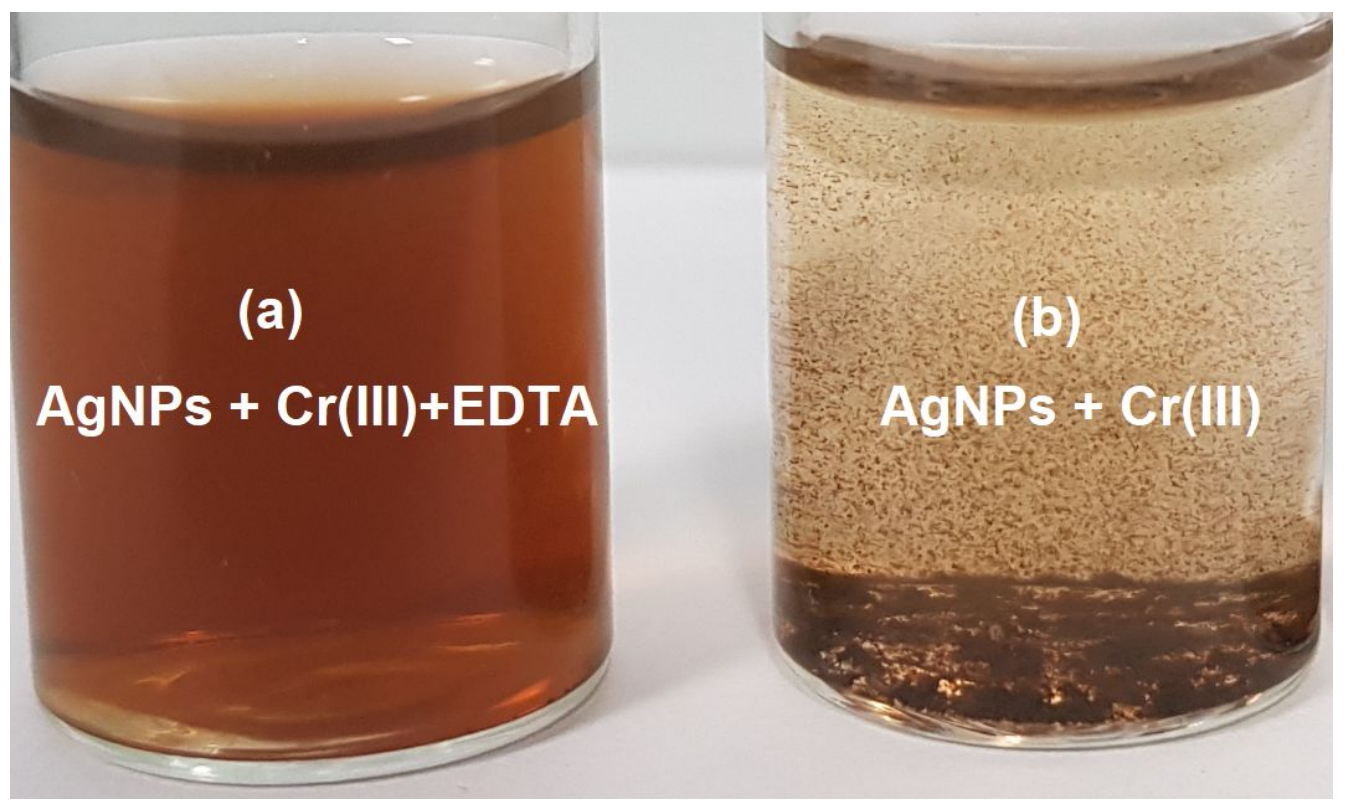

Figure S8 Dispersions of AgNPs in presence of (a) Cr(III)+EDTA or (b) only Cr(III). No significant precipitation was observed by bare eyes before 12 hours of mixing with AgNPs. However, precipitation occurred after 2 hours only when the medium contains $\mathrm{Cr}(\mathrm{III})$ in absence of EDTA. The photos were taken by the authors. 